\title{
An Anisotropic p-Adaptation Multigrid Scheme for Discontinuous Galerkin Methods
}

\author{
Andrés M. Rueda-Ramírez, Gonzalo Rubio, Esteban Ferrer, \\ and Eusebio Valero
}

\section{Introduction}

In recent decades, high-order discontinuous Galerkin (DG) methods have been gaining increasing popularity for high-accuracy solutions of systems of conservation laws, such as the compressible Euler and Navier-Stokes equations [5, 6, 22]. The lack of a continuity constraint on element interfaces makes DG methods robust for describing advection-dominated problems when an appropriate Riemann solver is selected $[5,12,22]$.

Multigrid methods speed up the iterative solution of large systems of equations using coarse-grid representations (lower levels). Iterative methods (known as smoothers in the multigrid community) are good at eliminating the high frequencies of the error fast; therefore, when applied to coarse-grid representations, they also reduce the low frequencies of the error. They have been broadly used in the highorder community in recent years in the form of p-multigrid $[2,8]$ (where levels are constructed using different polynomial orders) and hp-multigrid [14, 21] (where both the order and size of the elements are changed). Two types of multigrid methods can be found in the literature: linear and nonlinear multigrid. In our work, we make use of the nonlinear multigrid scheme, also known as the Full Approximation Scheme (FAS), since it enables the estimation of the truncation error of coarse representations, as will be shown. The smoother can be either a time-marching scheme (implicit or explicit), or an iterative method applied to the linearized problem.

\footnotetext{
A. M. Rueda-Ramírez $(\varangle) \cdot$ G. Rubio $\cdot$ E. Ferrer $\cdot$ E. Valero ETSIAE-UPM (School of Aeronautics - Universidad Politécnica de Madrid), Plaza Cardenal Cisneros, Madrid, Spain

e-mail: am.rueda@upm.es; g.rubio@upm.es; esteban.ferrer@upm.es; eusebio.valero@upm.es
} 
Because of the allowed discontinuities on element interfaces, DG methods are capable of handling non-conforming meshes with hanging nodes and/or different polynomial orders efficiently $[7,13,15]$. It is possible to take advantage of this feature to accelerate the computations through local adaptation strategies. Local adaptation can be performed by subdividing or merging elements (h-adaptation) or by enriching or reducing the polynomial order in certain elements ( $p$-adaptation). The main idea behind these methodologies is to reduce the number of degrees of freedom (NDOF) while maintaining a high accuracy, which translates into shorter computational times and reduced storage requirements. Furthermore, since several 2D and 3D implementations of the DG methods use tensor-product basis functions, it is possible to adapt the polynomial order in each coordinate direction independently. In order to identify the localized regions that need increased or decreased accuracy, an error estimator is commonly used.

There are several approaches to estimate the error and drive an adaptation method. In this work, we focus on truncation error estimates since it has been shown that a reduction of the truncation error controls the numerical accuracy of all functionals [10], hence reducing the truncation error necessarily leads to a more accurate lift and drag. The $\tau$-estimation method [4] is a way to estimate the truncation error locally that has been used to drive mesh adaptation strategies in low-order [9, 20] and high-order methods $[10,17,18]$. The adaptation strategy consists in converging a high order representation (reference mesh) to a specified global residual and then performing a single error estimation followed by a corresponding mesh adaptation process. Rueda-Ramírez et al. [19] developed a new method for estimating the truncation error of anisotropic representations that is cheaper to evaluate than previous implementations, and showed that it produces very accurate extrapolations of the truncation error, which enables the use of coarser reference meshes.

In this work, we employ the anisotropic truncation error estimator developed in [19] and the anisotropic p-adaptation method detailed in [18] to accelerate the computation of the compressible steady viscous flow past a NACA0012 at angle of attack $5^{\circ}, \operatorname{Re}_{\infty}=200$ based on the airfoil chord, and $M_{\infty}=0.2$. This particular settings correspond to a steady laminar flow, but the proposed method can be directly used with any steady solution (e.g. RANS). The paper is organized as follows: In Sect. 2, we briefly describe the methods used in this paper. In Sect. 3, we compare the performance of the proposed methods with traditional strategies for solving the flow past a NACA0012 and show the speed-up advantages for different accuracies. Finally, the conclusions are summarized in Sect. 4.

\section{Methods}

\subsection{DG Method}

We consider the approximation of systems of conservation laws,

$$
\partial_{t} \mathbf{q}+\nabla \cdot \mathscr{F}=\mathbf{s},
$$


where $\mathbf{q}$ is the vector of conserved variables, $\mathscr{F}$ is the flux dyadic tensor, and $\mathbf{s}$ is a source term. The domain $\Omega$ is partitioned in a mesh $\mathcal{T}=\{e\}$ consisting of $K$ non-overlapping elements $\Omega^{e}$. Multiplying equation (1) by a test function $\mathbf{v}$ and integrating by parts over each subdomain $\Omega^{e}$ yields the weak formulation:

$$
\int_{\Omega^{e}} \partial_{t} \mathbf{q v} \mathrm{d} \Omega^{e}-\int_{\Omega^{e}} \mathscr{F} \cdot \nabla \mathbf{v} \mathrm{d} \Omega^{e}+\int_{\partial \Omega^{e}} \mathscr{F} \cdot \mathbf{n v d} \sigma^{e}=\int_{\Omega^{e}} \mathbf{s v d} \Omega^{e} .
$$

Let $\mathbf{q}, \mathbf{s}, \mathscr{F}$ and $\mathbf{v}$ be approximated by piece-wise polynomial functions defined in the space of $L^{2}$ functions: $\mathscr{V}^{N}=\left\{\mathbf{v}^{N} \in L^{2}\left(\Omega^{e}\right):\left.\mathbf{v}^{N}\right|_{\Omega^{e}} \in \mathscr{P}^{N}\left(\Omega^{e}\right) \forall \Omega^{e} \in \mathscr{T}\right\}$, where $\mathscr{P}^{N}\left(\Omega^{e}\right)$ is the space of polynomials of degree at most $N$. The functions in $\mathcal{H}^{N}$ can be represented in each element as a linear combination of basis functions $\phi_{i}^{N} \in \mathscr{P}^{N}\left(\Omega^{e}\right)\left(\right.$ e.g. $\left.\left.\mathbf{q}^{N}\right|_{\Omega^{e}}=\sum_{i} \mathbf{Q}_{i}^{N} \phi_{i}^{N}\right)$, where $\phi_{i}^{N}$ are usually tensor product expansions. After some manipulations, the discontinuous Galerkin finite element discretization system is obtained:

$$
[\underline{\mathbf{M}}] \partial_{t} \mathbf{Q}^{N}+\mathbf{F}\left(\mathbf{Q}^{N}\right)=[\underline{\mathbf{M}}] \mathbf{S}^{N},
$$

where $[\underline{\mathbf{M}}]$ is the mass matrix and $\mathbf{F}$ is a nonlinear operator, which are the assembled global versions of the element-wise mass matrices and nonlinear operators:

$$
\begin{aligned}
\underline{\underline{\mathbf{M}}}]_{i, j}^{e} & =\int_{\Omega^{e}} \phi_{i} \phi_{j} \mathrm{~d} \Omega^{e} \\
\mathbf{F}^{e}(\mathbf{Q})_{j} & =\sum_{i=1}^{\mathrm{NDOF}^{e}}\left[-\int_{\Omega^{e}} \mathscr{F}_{i}^{e} \cdot \phi_{i} \nabla \phi_{j} \mathrm{~d} \Omega^{e}\right]+\int_{\partial \Omega^{e}} \mathscr{F}^{* N}\left(\mathbf{Q}, \mathbf{Q}^{-}, \mathbf{n}\right) \phi_{j} \mathrm{~d} \sigma^{e},
\end{aligned}
$$

where $\mathscr{F}_{i}^{e}$ is the $i$ th position of the vector $\mathscr{F}^{e}$, which contains the value of $\mathscr{F}^{e}$ for all the degrees of freedom of element $e$. In the rest of this paper, bold uppercase Roman letters and bold Greek letters are used to note vectors spanning several degrees of freedom, unless specified.

The numerical flux function $\mathscr{F}^{*}$ allows to uniquely define the flux at the element interfaces and to weakly prescribe the boundary data as a function of the conserved variable on both sides of the boundary/interface and the normal vector. In the present work, we use the scheme by Roe [16] as the advective Riemann solver and the original scheme by Bassi and Rebay [1] (BR1) as the diffusive Riemann solver.

\subsection{Full Approximation Scheme p-Multigrid}

The Full Approximation Scheme (FAS) is a nonlinear version of the multigrid method that is specially suited to solve systems of nonlinear equations [4]. Departing from Eq. (3) and defining the operator $\mathbf{A}\left(\mathbf{Q}^{N}\right)=[\underline{\mathbf{M}}]^{-1} \mathbf{F}\left(\mathbf{Q}^{N}\right)$, the steady-state 
problem of order $P$ yields

$$
\mathbf{A}\left(\mathbf{Q}^{P}\right)=\mathbf{S}^{P}
$$

After $\beta_{1}$ sweeps of a smoother, a non-converged solution $\tilde{\mathbf{Q}}^{P}$ is obtained that has an associated discretization error $\boldsymbol{\epsilon}^{P}=\mathbf{Q}^{P}-\tilde{\mathbf{Q}}^{P}$. The FAS multigrid procedure consists in obtaining an approximation to the discretization error in a coarse grid of order $N$ and projecting it to the original problem of order $P$ :

$$
\boldsymbol{\epsilon}^{P}=\underline{\mathbf{I}}_{N}^{P} \boldsymbol{\epsilon}^{N}=\underline{\mathbf{I}}_{N}^{P}\left(\mathbf{Q}^{N}-\underline{\mathbf{I}}_{P}^{N} \tilde{\mathbf{Q}}^{P}\right),
$$

where $\underline{\mathbf{I}}_{N}^{P}$ is an $L^{2}$ projection operator $N \rightarrow P$ and $\mathbf{Q}^{N}$ is the solution to the coarsegrid problem:

$$
\mathbf{A}^{N}\left(\mathbf{Q}^{N}\right)=\mathbf{S}^{N},
$$

where the source term is defined as

$$
\mathbf{S}^{N}=\mathbf{A}^{N}\left(\underline{\mathbf{I}}_{P}^{N} \tilde{\mathbf{Q}}^{P}\right)+\underline{\mathbf{I}}_{P}^{N}\left(\mathbf{S}^{P}-\mathbf{A}^{P}\left(\tilde{\mathbf{Q}}^{P}\right)\right) .
$$

In practice, several p-multigrid levels are used in V- or W-cycles. The smoothing steps that are performed when coarsening are called pre-smoothing sweeps, and the ones performed when refining back are called post-smoothing sweeps. Furthermore, $\mathbf{Q}^{N}$ is not obtained exactly in the coarse grids, but approximated using an iterative method $\tilde{\mathbf{Q}}^{N} \rightarrow \mathbf{Q}^{N}$. In this work, we use a third order low-storage Runge-Kutta (RK3) as the smoother and V-cycles.

\section{$2.3 \tau$-Based p-Adaptation}

In this section we show how to drive an anisotropic p-adaptation procedure using the truncation error, which is estimated in the multigrid procedure.

\subsubsection{The Anisotropic $\tau$-Estimation Method}

The non-isolated truncation error of a discretization of order $N$ is defined as

$$
\tau^{N}=\mathcal{R}^{N}\left(\mathbf{I}^{N} \mathbf{q}\right)-\mathcal{R}(\mathbf{q}),
$$

where $\mathbf{q}$ is the exact solution to the problem, $\mathbf{I}^{N}$ is a discretizing operator, $\mathcal{R}$ is the continuous partial differentiation operator, and $\mathcal{R}^{N}$ is the discrete partial 
differentiation operator. From Eqs. (1) and (3):

$$
\begin{gathered}
\mathcal{R}(\mathbf{q})=\mathbf{s}-\nabla \cdot \mathscr{F}, \\
\mathcal{R}^{N}\left(\boldsymbol{I}^{N} \mathbf{q}\right)=[\underline{\mathbf{M}}] \mathbf{S}^{N}-\mathbf{F}\left(\boldsymbol{I}^{N} \mathbf{q}\right),
\end{gathered}
$$

where $\boldsymbol{I}^{N}$ is an operator that samples the exact solution on the points that correspond to the degrees of freedom of a representation of order $N$, and therefore Eq. (12) corresponds to the sampled values of $\mathcal{R}^{N}\left(\mathbf{I}^{N} \mathbf{q}\right)$.

Note that in steady cases, $\mathcal{R}(\mathbf{q})=0$ holds. Since the exact solution $\mathbf{q}$ is usually not at hand, we utilize the quasi a-piori $\tau$-estimation method, which approximates the exact solution with the non-converged solution on a high-order grid $\mathbf{q} \approx \tilde{\mathbf{q}}^{P}$, where $N<P$. Therefore, the steady non-isolated truncation error estimation yields

$$
\tau_{P}^{N}=\mathcal{R}^{N}\left(\underline{\mathbf{I}}_{P}^{N} \tilde{\mathbf{q}}^{P}\right) \rightarrow \boldsymbol{\tau}_{P}^{N}=\mathcal{R}^{N}\left(\underline{\mathbf{I}}_{P}^{N} \tilde{\mathbf{Q}}^{P}\right)=[\underline{\mathbf{M}}] \mathbf{S}^{N}-\mathbf{F}\left(\underline{\mathbf{I}}_{P}^{N} \tilde{\mathbf{Q}}^{P}\right) .
$$

On the left side of the arrow is the estimation of the truncation error that lives in the space $\mathscr{y}^{N}$, and on the right side is the sampled form of the truncation error estimation on the points that correspond to the degrees of freedom. In a DG representation, one can also define the isolated truncation error $\hat{\tau}$ as

$$
\hat{\boldsymbol{\tau}}_{P}^{N}=\hat{\boldsymbol{\mathcal { R }}}^{N}\left(\underline{\mathbf{I}}_{P}^{N} \tilde{\mathbf{Q}}^{P}\right)=[\underline{\mathbf{M}}] \mathbf{S}^{N}-\hat{\mathbf{F}}\left(\underline{\mathbf{I}}_{P}^{N} \tilde{\mathbf{Q}}^{P}\right),
$$

where $\hat{\mathbf{F}}$ is the assembled version of the isolated nonlinear operator, defined elementwise as

$$
\mathbf{F}^{e}(\mathbf{Q})_{j}=\sum_{i=1}^{\mathrm{NDOF}^{e}}\left[-\int_{\Omega^{e}} \mathscr{F}_{i}^{e} \cdot \phi_{i} \nabla \phi_{j} \mathrm{~d} \Omega^{e}\right]+\int_{\partial \Omega^{e}} \mathscr{F}^{N} \cdot \mathbf{n} \phi_{j} \mathrm{~d} \sigma^{e} .
$$

Note that Eq. (15) is (5) without substituting $\mathscr{F}$ by the numerical flux $\mathscr{F}^{*}$. This change eliminates the influence of the neighboring elements and boundaries on the truncation error of each element. We drop the hat notation in the next statements since they are valid for both the isolated and non-isolated truncation error.

The $\tau$-estimation method can also be used with anisotropic representations, i.e.

$$
\tau_{P_{1} P_{2}}^{N_{1} N_{2}}=\mathcal{R}^{N_{1} N_{2}}\left(\underline{\mathbf{I}}_{P_{1} P_{2}}^{N_{1} N_{2}} \tilde{\mathbf{q}}^{P_{1} P_{2}}\right),
$$

where $N_{i}$ and $P_{i}$ are the polynomial orders in the direction $i$ of the analyzed representation and the high-order reference solution, respectively, where $N_{i}<P_{i}$. Additionally, Rueda-Ramírez et al. [19] showed that the truncation error of an anisotropic representation can be estimated using directional components:

$$
\tau^{N_{1} N_{2}} \approx \tau_{1}^{N_{1} N_{2}}+\tau_{2}^{N_{1} N_{2}} \approx \tau_{P_{1} P_{2}}^{N_{1} P_{2}}+\tau_{P_{1} P_{2}}^{P_{1} N_{2}}
$$


where the directional components in discrete form are therefore,

$$
\boldsymbol{\tau}_{1}=\boldsymbol{\tau}_{P_{1} P_{2}}^{N_{1} P_{2}}=[\underline{\mathbf{M}}] \mathbf{S}^{N_{1} P_{2}}-[\underline{\mathbf{M}}] \mathbf{A}\left(\underline{\mathbf{I}}_{P_{1} P_{2}}^{N_{1} P_{2}} \tilde{\mathbf{Q}}^{P_{1} P_{2}}\right),
$$

and that these directional components decrease exponentially with the polynomial order in smooth solutions. Consequently, it is possible to use a semi-converged solution $\tilde{\mathbf{q}}^{P_{1} P_{2}}$ to estimate $\tau^{N_{1} N_{2}}\left(N_{i}<P_{i}\right)$ and then extrapolate the directional components $\tau_{i}$ to obtain the values of $\tau^{N_{1} N_{2}}$ for $N_{i}>P_{i}$. Figure 1a shows a graphical representation of the truncation error $\tau^{N_{1} N_{2}}$ as estimated with a semiconverged solution of order $P_{1}=P_{2}=5$.

\subsubsection{The p-Adaptation Multigrid Scheme}

It has been shown that the use of FAS p-multigrid methods speeds up the computation of steady-state and unsteady solutions of the compressible NavierStokes equations [2, 8]. In addition, Rueda-Ramírez et al. [18] showed that the truncation error of an anisotropic representation can be inexpensively obtained inside an anisotropic p-multigrid cycle that performs the coarsening in one coordinate direction at a time. In fact, the second term of Eq. (18) is naturally computed in an anisotropic multigrid for obtaining the coarse-grid source term (Eq. (9)).

Therefore, we propose a p-adaptation multigrid scheme that makes use of the multigrid as a solver, but also as an error estimator. Every time the error is estimated, an anisotropic p-multigrid strategy is used to generate a truncation error map for each element, like the one in Fig. 1a. Afterwards, the polynomial orders in the different coordinate directions are selected for

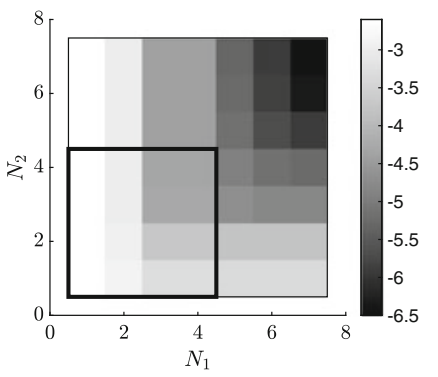

(a)

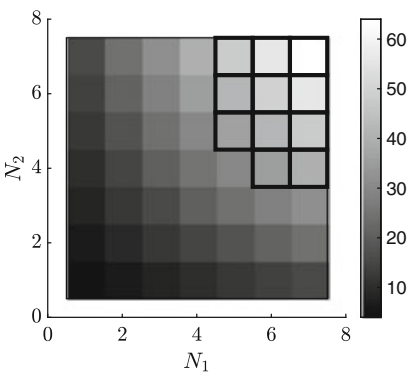

(b)

Fig. 1 (a) Truncation error map for a specific element that shows $\log \left\|\tau_{5,5}^{N_{1} N_{2}}\right\|_{\infty}$ as a function of $N_{1}$ and $N_{2}$ (the black box shows the limit between the estimated and extrapolated maps). (b) Map of degrees of freedom (the black boxes show the polynomial orders that achieve $\left\|\tau_{5,5}^{N_{1} N_{2}}\right\|_{\infty}<$ $10^{-5}$ ) 
each element, such that a truncation error threshold $\tau_{\max }$ is achieved with the minimum NDOF possible, as illustrated in Fig. 1b. In the simulations shown in this paper, the reference representation, $\tilde{\mathbf{q}}^{P}$, is converged to a residual $\tau_{\text {max }} / 10$ before the p-adaptation stage, so that the truncation error is accurately estimated down to $\tau_{\max }$, as was shown necessary by Kompenhans et al. [10].

\section{Flow Past a NACA0012 Airfoil}

In this section, we compare the performance of the proposed p-adaptation multigrid scheme with a uniformly adapted p-multigrid method (without local padaptation) and a uniformly adapted RK3 method when solving the steady viscous flow past a NACA0012 airfoil at angle of attack $5^{\circ}, \operatorname{Re}_{\infty}=200\left(L_{\infty}=\right.$ $L_{\text {chord}}$ ) and $\mathrm{M}_{\infty}=0.2$. This particular settings correspond to a steady laminar flow, but the proposed method can be directly used with any steady solution (e.g. RANS). An unstructured mesh of 2011 quadrilateral elements is employed (Fig. 2).

In the cases where multigrid is employed, the RK3 scheme is used as the iterative method (smoother), so that additional speed-ups are only due to the methods exposed in Sect.2. As in [18], a residual-based smoothing strategy is performed. The minimum number of smoothing sweeps is $\beta=200$ for the coarsest multigrid level $(N=1)$ and $\beta=50$ for any other level. After every $\beta$ presmoothing sweeps, the residual in the next (coarser) representation is checked. If $\left\|\mathcal{R}^{N}\right\|_{\infty}<1.2\left\|\mathcal{R}^{N-1}\right\|_{\infty}$, the pre-smoothing is stopped; otherwise, $\beta$ additional

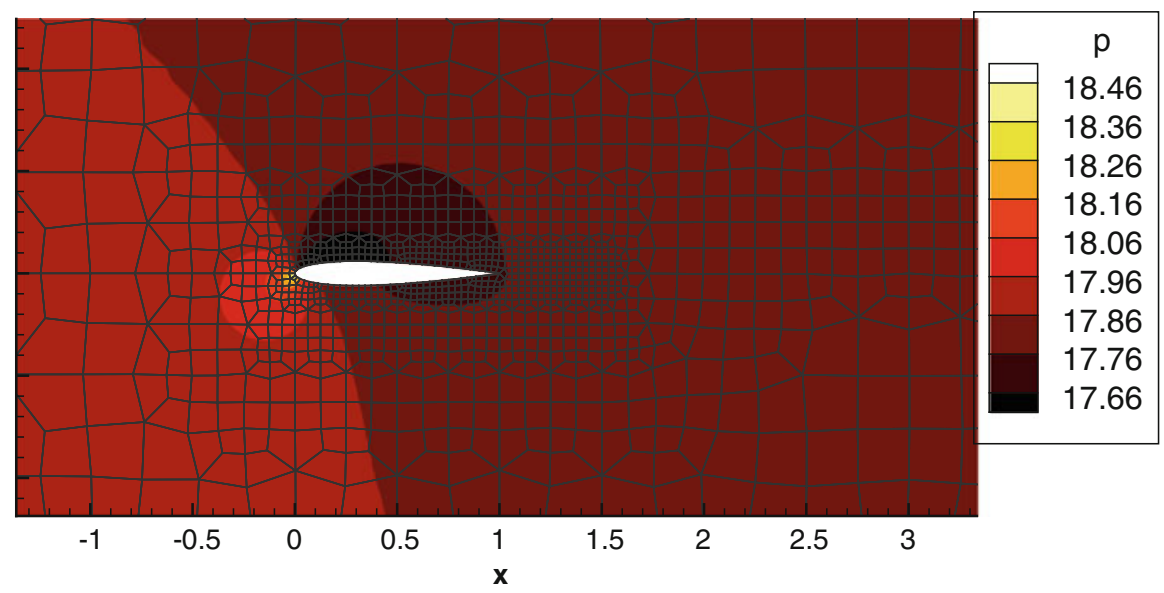

Fig. 2 Pressure contours of the flow past a NACA0012 at angle of attack $5^{\circ}$ 
sweeps are performed. Similarly, the norm of the residual after the post-smoothing is forced to be at least as low as it was after the pre-smoothing, $\left\|\mathcal{R}_{\text {post }}^{N}\right\|_{\infty} \leq$ $\left\|\mathcal{R}_{\text {pre }}^{N}\right\|_{\infty}$. If that condition is not fulfilled, additional $\beta$ sweeps are taken until it is.

The isolated truncation error estimate is used to drive the p-adaptation method since it has been shown to provide better results than the non-isolated one [1719]. The conservative form (Eq. (1)) of the compressible Navier-Stokes equations is discretized using the Discontinuous Galerkin Spectral Element Method (DGSEM) $[3,12]$, which is a nodal (collocation) version of a DG method that uses Gauss points as the solution nodes and quadrature points, obtaining diagonal mass matrices. However, the methods that are exposed here can be applied to any DG scheme with tensor-product basis functions.

In [18] it was explained that, when using the DGSEM in general 3D curved meshes and p-nonconforming representations, the order of the mapping must be at most $M \leq N / 2$ for the numerical representation to be free-stream preserving. For this reason, the use of a conforming algorithm was proposed, which forces the polynomial orders to be conforming in the first layer of elements on a curved boundary. The use of a conforming algorithm is necessary to retain the well-known $M \leq N$ condition of the DGSEM [11]. In this work, we use the conforming algorithm on the airfoil surface since it showed to produce better results, although its use is not imperative as the considered test case is 2D.

For the uniformly adapted cases, the polynomial order is varied between $N=2$ and $N=7$. For the cases with local p-adaptation, a single-stage anisotropic p-adaptation procedure is performed, and the minimum polynomial order after adaptation is set to $N_{\min }=1$, whereas the maximum polynomial order after adaptation is set to $N_{\max }=7$. The relative drag and lift errors of the adapted meshes are assessed by comparing with a reference solution of order $N=8$ :

$$
e_{\text {drag }}^{N=8}=\frac{\left|C_{d}-C_{d}^{N=8}\right|}{C_{d}^{N=8}}, e_{\text {lift }}^{N=8}=\frac{\left|C_{d}-C_{l}^{N=8}\right|}{C_{l}^{N=8}} .
$$

Figure 3 shows a comparison between the errors obtained using the $\hat{\tau}$-based adaptation procedure and the ones using uniform p-refinement. As can be observed, the number of degrees of freedom is substantially reduced for the same accuracy when using the $\hat{\tau}$-based p-adaptation. This reduction translates into a reduction of the CPU-times. It is interesting to point out that, as the isolated truncation error threshold $\hat{\tau}_{\text {max }}$ is decreased, the polynomial orders of the mesh tend to the maximum specified polynomial order, $N_{\max }=7$. Consequently, the lift and drag coefficients also tends to $C_{l}^{N=7}$. Using Fig. 3, it is possible to compute a speed-up for different levels of accuracy. Table 1 summarizes the speed-up calculations for the maximum level of accuracy that was achieved for the drag and lift coefficients. 


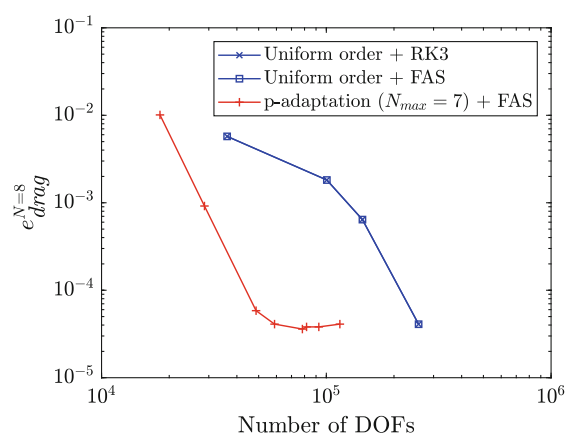

(a)

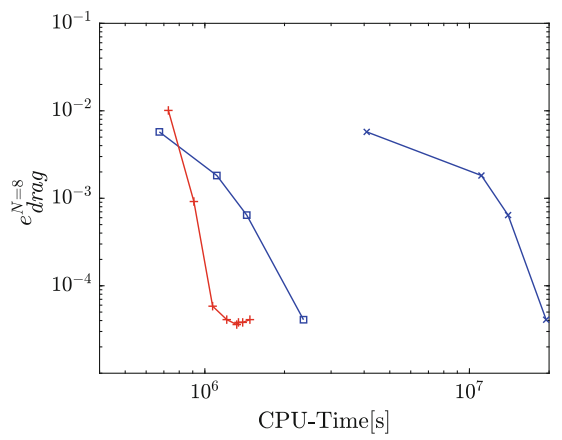

(c)

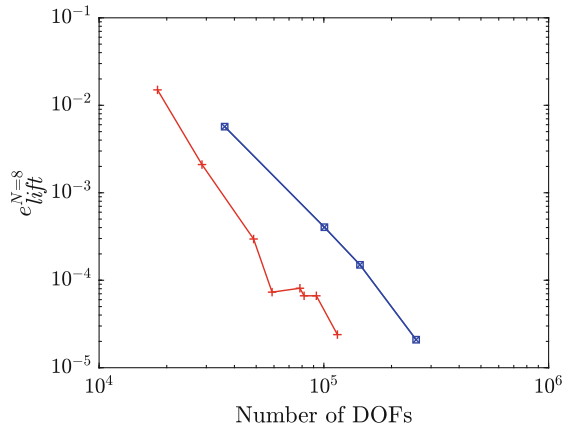

(b)

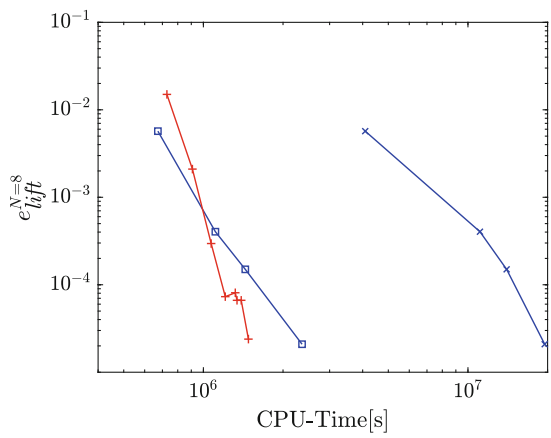

(d)

Fig. 3 Relative error in the drag and lift coefficients for different methods for the flow past the NACA0012 airfoil. The blue lines represent uniform refinement, and the red lines represent the $\hat{\tau}$-based p-adaptation procedure with $N_{\max }=7$. (a) Drag error vs. DOFs; (b) lift error vs. DOFs; (c) drag error vs. CPU-time; (d) lift error vs. CPU-time

Table 1 Computation times and speed-up for the different methods after converging until $\|\mathbf{r}\|_{\infty}<$ $10^{-9}$

\begin{tabular}{l|l|r|c|l|l|c}
\hline \multirow{2}{*}{ Method } & \multicolumn{4}{|l|}{ Drag coefficient $\left(e_{\text {drag }} \leq \times 4.1 \times 10^{-5}\right)$} & \multicolumn{3}{l}{ Lift coefficient $\left(e_{\text {lift }} \leq 2.4 \times 10^{-5}\right)$} \\
\cline { 2 - 7 } & CPU-time [s] & Time [\%] & Speed-up & CPU-time [s] & Time [\%] & Speed-up \\
\hline RK3 & $1.95 \times 10^{7}$ & $100.00 \%$ & 1.00 & $1.95 \times 10^{7}$ & $100.00 \%$ & 1.00 \\
\hline FAS & $2.36 \times 10^{6}$ & $12.10 \%$ & 8.26 & $2.36 \times 10^{6}$ & $12.10 \%$ & 8.26 \\
\hline $\begin{array}{l}\text { FAS + p- } \\
\text { adaptation }\end{array}$ & $1.21 \times 10^{6}$ & $6.20 \%$ & 16.13 & $1.48 \times 10^{6}$ & $7.58 \%$ & 13.19 \\
\hline
\end{tabular}

Figure 4 shows the distribution of polynomial orders after the single-stage adaptation procedure for a threshold of $\tau_{\max }=5 \times 10^{-4}$, which has related errors of $e_{\text {drag }}^{N=8}=4.10 \times 10^{-5}$ and $e_{\text {lift }}^{N=8}=7.31 \times 10^{-5}$. As can be observed, the elements that are enriched are mainly the ones on the boundary layer (specially leading and trailing edge), and the zones of the wake where the element size changes significantly. 


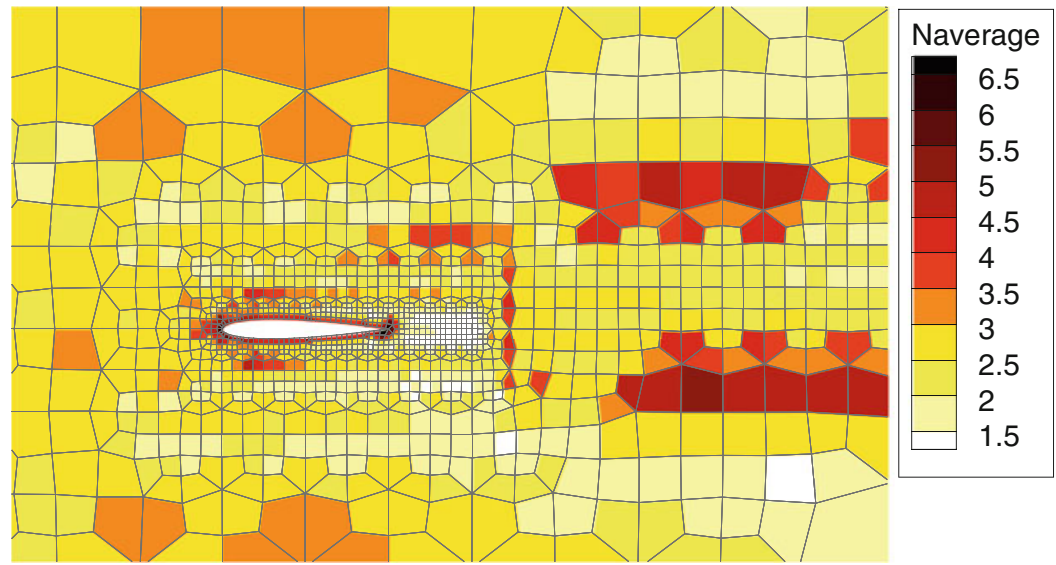

Fig. 4 Polynomial order distribution after the anisotropic p-adaptation. $N_{\text {average }}=\left(N_{1}+N_{2}\right) / 2$

\section{Conclusions}

In this work, we have applied recently developed error estimators and anisotropic p-adaptation methods in conjunction with multigrid solving strategies for solving the compressible Navier-Stokes equations. In particular, we have shown that the coupling of anisotropic truncation error-based p-adaptation methods with $\mathrm{p}$ multigrid schemes can speed up the computation of steady-state solutions of PDEs. The achieved speed-up depends on the desired accuracy, being this method optimal when high accuracy is required (low errors). In particular, a speed-up of 16.13 was achieved for the computation of the steady compressible viscous flow past a NACA0012 airfoil at angle of attack $5^{\circ}$ with respect to the uniformly adapted representation without multigrid.

Acknowledgements This project has received funding from the European Union's Horizon 2020 Research and Innovation Program under the Marie Skłodowska-Curie grant agreement No 675008 for the SSeMID project. The authors acknowledge the computer resources and technical assistance provided by the Centro de Supercomputación y Visualización de Madrid (CeSViMa).

\section{References}

1. Bassi, F., Rebay, S.: A high-order accurate discontinuous finite element method for the numerical solution of the compressible Navier-Stokes equations. J. Comput. Phys. 131, 267279 (1997)

2. Bassi, F., Ghidoni, A., Rebay, S., Tesini, P.F.: High-order accurate p-multigrid discontinuous Galerkin solution of the Euler equations. Int. J. Numer. Methods Fluids 60, 847-865 (2009)

3. Black, K.: A conservative spectral element method for the approximation of compressible fluid flow. Kybernetika 35, 133-146 (1999) 
4. Brandt, A., Livne, O.E.: Multigrid Techniques: 1984 Guide with Applications to Fluid Dynamics, Revised Edition. SIAM, Philadelphia (2011)

5. Cockburn, B., Shu, C.-W.: The local discontinuous Galerkin method for time-dependent convection-diffusion systems. SIAM J. Numer. Anal. 35, 2440-2463 (1998)

6. Ferrer, E.: An interior penalty stabilised incompressible discontinuous Galerkin-Fourier solver for implicit large eddy simulations. J. Comput. Phys. 348, 754-775 (2017)

7. Ferrer, E., Willden, R.H.: A high order Discontinuous Galerkin-Fourier incompressible 3D Navier-Stokes solver with rotating sliding meshes. J. Comput. Phys. 231, 7037-7056 (2012)

8. Fidkowski, K.J., Oliver, T.A., Lu, J., Darmofal, D.L.: p-Multigrid solution of high-order discontinuous Galerkin discretizations of the compressible Navier-Stokes equations. J. Comput. Phys. 207, 92-113 (2005)

9. Fraysse, F., Rubio, G., De Vicente, J., Valero, E.: Quasi-a priori mesh adaptation and extrapolation to higher order using $\tau$-estimation. Aerosp. Sci. Technol. 38, 76-87 (2014)

10. Kompenhans, M., Rubio, G., Ferrer, E., Valero, E.: Adaptation strategies for high order discontinuous Galerkin methods based on Tau-estimation. J. Comput. Phys. 306, 216-236 (2016)

11. Kopriva, D.A.: Metric identities and the discontinuous spectral element method on curvilinear meshes. J. Sci. Comput. 26, 301-327 (2006)

12. Kopriva, D.: Implementing Spectral Methods for Partial Differential Equations: Algorithms for Scientists and Engineers. Springer, Berlin (2009)

13. Kopriva, D.A., Woodruff, S.L., Hussaini, M.Y.: Computation of electromagnetic scattering with a non-conforming discontinuous spectral element method. Int. J. Numer. Methods Eng. 53, 105-122 (2002)

14. Mitchell, W.F., Division, C.S.: The hp -multigrid method applied to hp -adaptive refinement of triangular grids. Numer. Linear Algebra Appl. 17, 211-228 (2010)

15. Rivière, B.: Discontinuous Galerkin Methods for Solving Elliptic and Parabolic Equations Theory and Implementation. SIAM, Philadelphia (2008)

16. Roe, P.L.: Approximate Riemann solvers, parameter vectors, and difference schemes. J. Comput. Phys. 43, 357-372 (1981)

17. Rubio, G., Fraysse, F., Kopriva, D.A., Valero, E.: Quasi-a priori truncation error estimation in the DGSEM. J. Sci. Comput. 64, 425-455 (2015)

18. Rueda-Ramírez, A.M., Manzanero, J., Ferrer, E., Rubio, G., Valero, E.: A p-multigrid strategy with anisotropic p-adaptation based on truncation errors for high-order discontinuous Galerkin methods. J. Comput. Phys. 378, 209-233 (2019)

19. Rueda-Ramírez, A.M., Rubio, G., Ferrer, E., Valero, E.: Truncation error estimation in the panisotropic discontinuous Galerkin spectral element method. J. Sci. Comput. 78(1), 433-466 (2018)

20. Syrakos, A., Efthimiou, G., Bartzis, J.G., Goulas, A.: Numerical experiments on the efficiency of local grid refinement based on truncation error estimates. J. Comput. Phys. 231, 6725-6753 (2012)

21. Wang, L., Mavriplis, D.: Adjoint-based h-p adaptive discontinuous Galerkin methods for the compressible Euler equations, J. Comput. Phys. 228, 7643-7661 (2009)

22. Wang, Z., Fidkowski, K., Abgrall, R., Bassi, F., Caraeni, D., Cary, A., Deconinck, H., Hartmann, R., Hillewaert, K., Huynh, H., Kroll, N., May, G., Persson, P.-O., van Leer, B., Visbal, M.: High-order CFD methods: current status and perspective. Int. J. Numer. Methods Fluids 72, 811-845 (2013) 
Open Access This chapter is licensed under the terms of the Creative Commons Attribution 4.0 International License (http://creativecommons.org/licenses/by/4.0/), which permits use, sharing, adaptation, distribution and reproduction in any medium or format, as long as you give appropriate credit to the original author(s) and the source, provide a link to the Creative Commons licence and indicate if changes were made.

The images or other third party material in this chapter are included in the chapter's Creative Commons licence, unless indicated otherwise in a credit line to the material. If material is not included in the chapter's Creative Commons licence and your intended use is not permitted by statutory regulation or exceeds the permitted use, you will need to obtain permission directly from the copyright holder. 